\title{
Distributed Implementation of Petri nets in Control Applications
}

\author{
Ramon Piedrafita, Danilo Tardioli and Jose Luis Villarroel \\ Department of Computer Science and Systems Engineering, University of Zaragoza \\ Spain
}

\section{Introduction}

In this chapter, we propose a platform for the implementation of distributed discrete event control systems. We further propose a new architecture for the distributed implementation of Petri nets (PN) in control applications. The architecture covers the local execution of PN and the communication among controllers. Both synchronous and asynchronous communication paradigms are supported. A framework of classes for supporting the realtime communication and real-time execution or remote calls has been developed. The Realtime Wireless Multi-hop Protocol has been adopted. With this protocol and the framework of classes developed, control deadlines of the system can be guaranteed. In order to ensure the access to common resources, a new class of communication places, the Auction Communication Places, is proposed. To support the real-time and concurrent characteristics, we have used the Java real-time specification. In order to implement PN, we have extended the concurrent coordinators technique, developed in previous works, to distributed systems introducing the distributed coordinators. To demonstrate the practical utility and feasibility of the architecture, we have applied it to the control of a flexible manufacturing cell.

A software implementation of a PN is a program that triggers the firing of the net transitions observing the marking evolution rules, that is, it plays the token game. Depending on the criteria, a Petri net implementation can be classified as compiled or interpreted, sequential or concurrent, and centralized or decentralized. An implementation is interpreted if the net structure and the marking are codified as data structures. The data structures are used by one or more tasks called interpreters to establish the net evolution. The interpreters do not depend on the implemented net. A compiled implementation is based on the generation of one or more tasks whose control flow corresponds to net evolutions.

In previous works (Piedrafita and Villarroel 2006 a) a centralized control architecture and a development environment based on Petri nets and Java language was developed. In the present work, this control architecture is extended to be applied to distributed control systems. In this work we develop the distributed implementation of PN. To achieve this, we have developed a new technique called distributed coordinators, and to solve conflicts between distributed processes we propose a new technique: the Auction Communication Place.

In this technique, the execution of the coordinators takes place in several hosts, connected by a real-time communication network. In each host one or more coordinators executes its Petri net. The coordinators read the inputs, execute its Petri net (the token player) and write the 
outputs, i.e. achieving local machine control. For the necessary information interchange between hosts, the implementation is completed using the necessary communication mechanisms.

The partition into two or more subnets leads to the appearance of shared places among subnets. These places are known as communication places. The communication places can be shared between Petri nets of the same host, or between Petri nets of different hosts. A communication place is implemented in one of the hosts and belongs to the PN executed in the machine in question.

A Middleware that allows the implementation of distributed PN has been developed. For this purpose, a set of Java Classes to implement synchronous and asynchronous communication paradigms has been designed. These classes implement the local and remote communications places, the auction communication place and the auction solver.

To implement the communication between the different coordinators, a protocol that supports real time traffic over Ethernet is needed. In our case the protocol RT-WMP (Tardioli andVillarroel 2007) developed at the University of Zaragoza, has been chosen.

The RT-WMP is a novel protocol that supports hard real-time traffic. In this sense in RTWMP, end-to-end messages delay has a bounded and known duration and it manages global static priorities of messages. The protocol has been designed to connect a relatively small group of nodes. It is based on a token-passing scheme. RT-WMP has a built-in error recovery mechanism that can recover from certain types of errors without jeopardizing the real-time behaviour. The RT-WMP can run over commercial hardware without modifications.

The practical application of the technologies presented in this paper were tested using a flexible manufacturing cell installed at the Department of Computer Science and Systems Engineering at the University of Zaragoza, for research and teaching purposes.

We have installed a new distributed control system based on several computers, connected by communications networks, and a fieldbus. One of the computers is equipped with a fieldbus master card, where the input/output modules of the machines are connected. The rest of computers control the input/output hardware by Industrial Ethernet.

This chapter is organized as follows: in Section 2, we review the different techniques used to implement PN. Section 3 describes distributed implementation of PN and in Section 4 the characteristics of the RT-WMP based implementation are indicated. The application over the flexible manufacturing cell is described in Section 5. In Section 6, we present conclusions and suggest future lines of research.

\section{Software Implementation of Petri Nets}

A Petri net is a graphical, mathematical tool that can be used to model discrete event systems (Murata 1989). PNs are bipartite graphs that have two types of nodes: places and transitions, which are represented by circles and bars respectively. Directional arcs connect places and transitions. Input arcs connect places with transitions, and output arcs connect transitions to places. Places contain a positive or null number of tokens. The token distribution over PN places is called marking and represents the system state. Transitions represent events. A transition firing models the occurrence of an event that changes the system state (the marking of the PN). 
In the last 25 years, researchers have devoted considerable attention to the software implementation of PN; see for example (Taubner 1988), (Bruno and Marchetto 1986), (Brams 1982), (Briz and Colom 1994), and (Colom, Silva et al. 1986). A software implementation is a program that triggers the firing of the net transitions observing the marking evolution rules, that is, it plays the token game. Depending on the criteria, a Petri net implementation can be classified as compiled or interpreted, sequential or concurrent, and centralized or decentralized. An implementation is interpreted if the net structure and the marking are codified as data structures. The data structures are used by one or more tasks called interpreters to establish the net evolution. The interpreters do not depend on the implemented net. A compiled implementation is based on the generation of one or more tasks whose control flow corresponds to net evolutions.

In a centralized implementation, the token player is executed by a single task, which is called the coordinator. To characterize a centralized implementation, the algorithm to determine which transitions are enabled and can fire is important. Apart from the simple exhaustive test of all of the transitions, there are various solutions for reducing the costs of the enabling test, including static or dynamic representing places (Colom, Silva et al. 1986) and transition-driven techniques (Briz and Colom 1994).
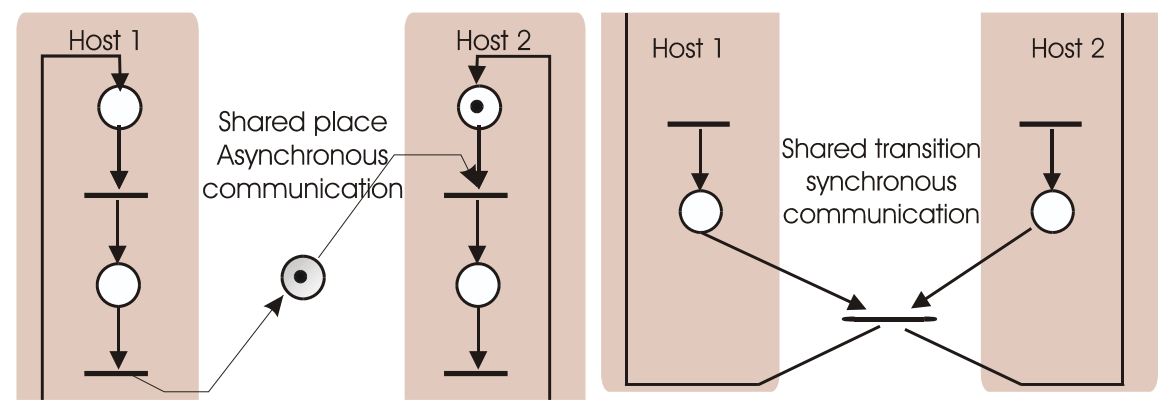

Fig. 1. Asynchronous and synchronous communication.

In a decentralized implementation, the net is decomposed into a set of sequential structures. Each of the structures is implemented by a task and the implementation is completed using the necessary communication and synchronization mechanisms.

In (Piedrafita and Villarroel 2006 a) we introduced centralized techniques into decentralized implementations, thereby creating a new technique called concurrent coordinators. The application can run several coordinators simultaneously by executing a sub-net for each subsystem. In this technique the net can be split following control or functional criteria, different from the behavioral ones needed for other decentralized techniques.

In this work we develop the distributed implementation of PN, extending the previous concurrent coordinators technique. We call this technique the distributed coordinators. In it, the execution of the coordinators takes place in several hosts, connected by a communication network. In each host one or more coordinators executes its Petri net. The coordinators read the inputs, executes its Petri net (the token player) and writes the outputs, i.e. achieving local machine control. For the necessary information interchange between hosts, the implementation is completed using the necessary communication mechanisms. 
A Middleware that allows the implementation of distributed PN has been developed. For this purpose, a set of Java Classes to implement synchronous and asynchronous communication paradigms has been designed. These classes implement the local and remote communications places, the auction communication place and the auction solver. To implement the communication between the different coordinators, a protocol is needed that supports real-time traffic over Ethernet. In our case the protocol RT-WMP has been chosen, but the same implementation could have been made with other real-time protocols such as RT-RMI (Borg and Wellings 2003) or RT-EP (Martínez, Harbour et al. 2003).

The classes for managing communication with the Real-time RT-WMP protocol have been developed. This set of classes also implements the necessary real-time execution of the remote procedure calls. Also, the classes for communicating with input/output modules in industrial field buses CANopen, Interbus and Industrial Ethernet have been developed.

We have implemented the distributed implementation techniques of $\mathrm{PN}$ in the Java language using the Java Real-time extension (Bollella and Gosling 2000) and following some ideas presented in (Piedrafita and Villarroel 2006 a) and (Piedrafita and Villarroel 2006 b). . The real-time extension provides Java with the necessary aspects for the programming of real-time systems, e.g., preemptive planning based on static priorities, asynchronous transfer of control, real-time high resolution clocks, and the possibility of execution over the Java garbage collector. In our implementations, we used the Real-time Java Virtual Machine JamaicaVM v2.7 (Aicas 2007). The target hardware was a personal computer with Pentium IV processor at $1.7 \mathrm{GHz}$, running Red Hat Linux 2.4.

\section{Distributed implementation of Petri nets}

In order to make a distributed implementation of $\mathrm{PN}$, a net partition has to be made. In previous works (Colom, Silva et al. 1986), (García and Villarroel 1998), (Villarroel 1990), the authors show how to make a Petri net partition in sequential processes, applying structural and behavioral analysis. In this work the partition is done following a functional criterion. The PNs are subdivided into several subnets with local access to the input/output modules criteria and the machine's local control criteria.

This partition sets out a set of subnets, a set of shared places and a set of shared transitions. The subnets can be implemented in several computers. The implementation of the subnets is made in a centralized form. In each computer one or more coordinators are in charge of executing the corresponding subnets. The coordinators are threads with real-time requirements. From the perspective of the Java Real-time Specification, they are periodic RealtimeThreads with high priority that are scheduled without round-robin.

The shared places model the asynchronous communications; the shared transitions model the synchronous communication. The communication between distributed PNs can be made in asynchronous form by means of shared places, and in synchronous form by means of shared transitions (see Fig. 1).

\subsection{Communication Places}

The partition into two or more subnets leads to the appearance of shared places among subnets. These places are known as communication places (see Fig. 2). A communication place is implemented as a protected object with synchronized methods to mark and unmark the place. Program 1 shows the communication place code. 
The communications places can be shared between Petri nets of the same host, or between Petri nets of different hosts. A communication place is implemented in one of the hosts and belongs to the PN executed in the machine in question.

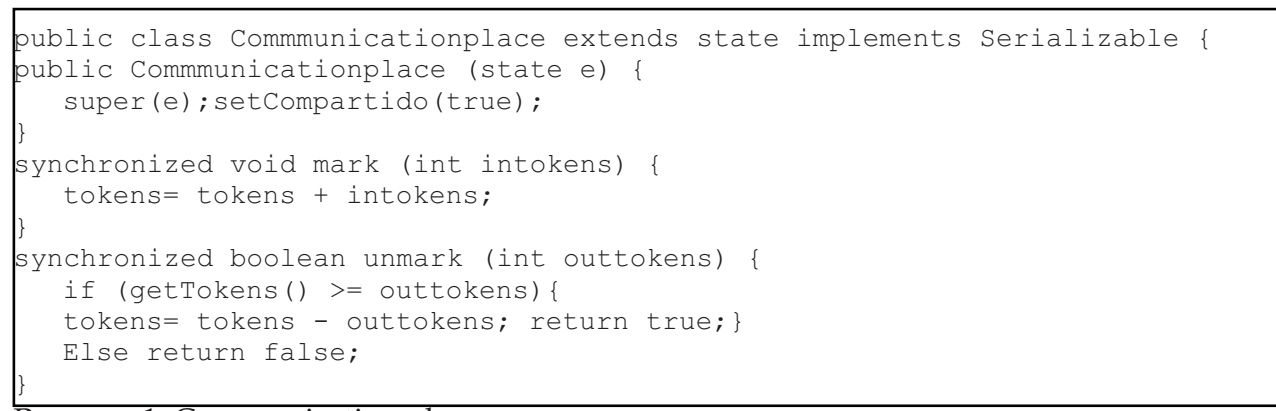

Program 1. Communication place

The interface of communication places is also implemented in the rest of the nodes that share it, as communication places remote interface. The calls to the methods of communication places remote interface are sent to communication places through the real-time communication network.

It is possible to access this place as:

- communication place, when the access is local in the host machine. The local coordinator and its communications places are implemented in the same Java Virtual machine. The Local coordinator access to its communication places callings is methods directly.

- communication place remote interface (CPRI), when the access is from another machine of the network. The CPRI is the remote implementation of the corresponding communication place. The CPRI and the remote coordinator are implemented in a remote host in its Java Virtual Machine. The Remote coordinator access to its CPRI callings is methods directly. These callings are directed to the communication places through the real-time communication network over the protocol RT-WMP.
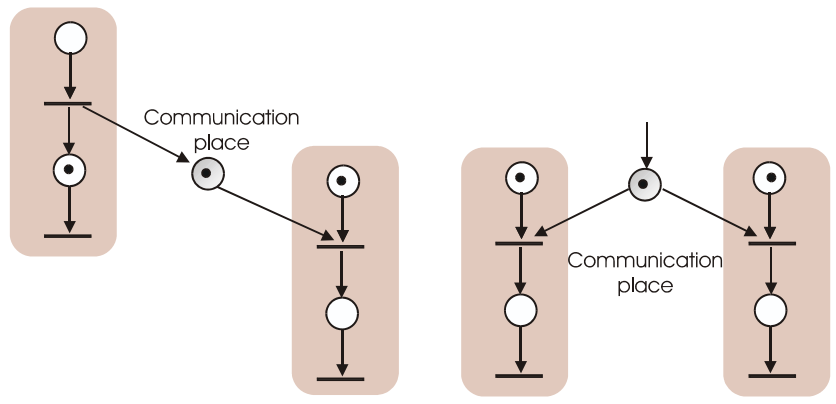

Fig. 2. Communication place: a) single destination b) multiple destination 


\subsection{Auction Communication Places}

If, in the execution of its Petri net, a coordinator needs to unmark a communication place, it is necessary to consult the number of available tokens and then unmark the communication place. This fact can cause problems since operation is not atomic. In our decentralized implementation (Piedrafita and Villarroel 2006 a), several coordinators, all of them implemented as Real-time Threads with the same priority, run simultaneously in a single computer. The execution is made in a single processor and threads are scheduled following a static priorities policy without round-robin. A coordinator can consult the number of tokens in a place and next fire the transition and unmark the place without pre-emption by another coordinator.

However, if implementation is distributed, the simultaneous execution of several coordinators takes place in several computers, and it can happen that several coordinators want to unmark a communication place simultaneously. These external conflicts can occur when a communication place has several output transitions distributed in different subnets. In this case, the conflict has been solved by means of the introduction of an auction where the transitions are fired in priority order. These shared places are implemented by means of a protected object called auction communication place.

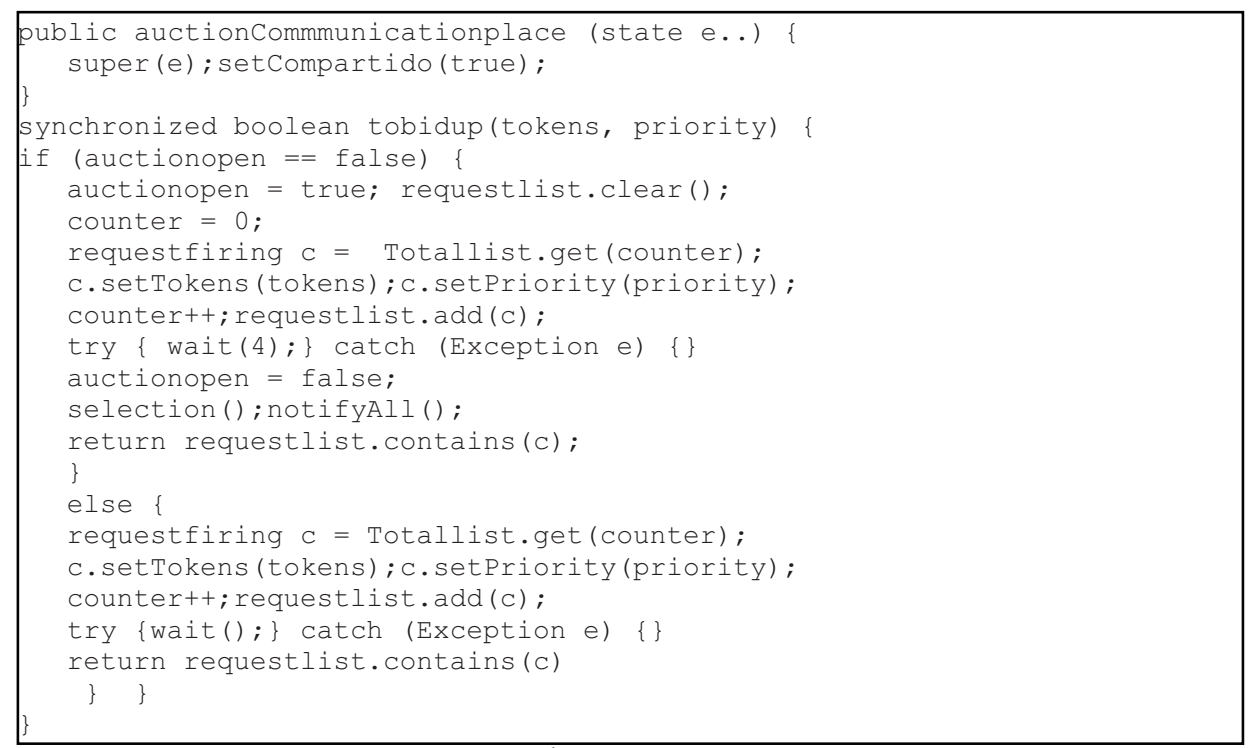

Program 2. Auction Communication Place

When a coordinator wants to unmark an auction communication place, it carries out a call to the method tobidup () of this one. The call is stored with its priority and the number of tokens requested. Next, if it is the first request, the caller (i.e. the first coordinator) is suspended for $4 \mathrm{~ms}$. Coordinators are implemented as Periodic Real-time Threads with 25 ms of period to obtain good control of the machines. The conflict must be solved within the $25 \mathrm{~ms}$ of period of the coordinators. During this $4 \mathrm{~ms}$, other local or remote coordinators can enter the auction to unmark that place. Their requests are stored and they are suspended by 
calling a wait() method. After $4 \mathrm{~ms}$, the first coordinator wakes up and closes the auction. Then it calls to the method selection() to assign tokens, and next with notifyall() wakes up the rest of the coordinators. If the assigned request list contains the request of the coordinator, the method tobidup () returns true. The execution of a tobidup() call consists of the execution of the test and the unmarking of the auction communication place. These two operations are made in atomic form.

In this way, the conflict is correctly solved, since all the transitions that want to unmark a place enter in the auction and the transitions are fired in priority order. With this method, remote coordinators can access the tokens. The maximum blocking time is $4 \mathrm{~ms}$ to avoid control problems, but also to allow the calls to tobidup() that travel through the real-time communication network to be taken into account.

In case of distributed simple conflicts where the communication places have several output transitions in the same PN, the implementation of the communication place must be made in an active object, in a thread. If several transitions that belong to the same PN wish to unmark the same communication place, the first call of tobidup () blocks the execution of the coordinator, thus the rest of transitions would not enter the auction. Therefore, to solve this problem, an active object that manages these situations is needed. We call it auction solver.

The auction solver receives requests from the output transitions, reserves tokens according to the priority and informs in a list which transitions can fire. When the suspend time is expired and the place is not unmarked by the transition, the auction solver cancels the reservation of the marks. The implementation of the auction solver is made up in a Real-time Thread of higher priority than the coordinator Thread.

\subsection{Synchronous Communication}

For the implementation of shared transitions in Java, it would be necessary to have some primitive of synchronous communication. Java does not have instructions that allow synchronous communication such as the ADA rendez-vous. Moreover, even if it were available, it could not be used since the rendez-vousconstruction blocks the caller thread. Therefore, the coordinator is blocked until the acceptance of the appointment and this involves the loss of control during that time. In order to implement the shared transitions in Java it is necessary to transform the PN according to Fig. 3. In this way, a synchronous communication using a shared transition is transformed into two asynchronous communications using communication places.

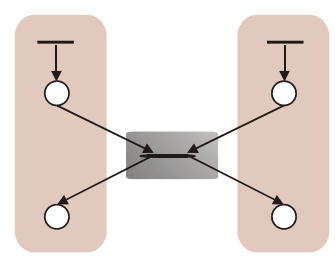

Fig. 3. Conversion of a synchronous communication into two asynchronous communications.

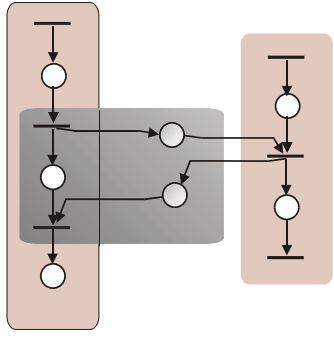

comunication into two asynchronous 


\section{Real-Time Communication}

In order to guarantee a bounded time response, real-time communication is necessary among the nodes of the PN, and the execution of the methods should be made with the appropriate real-time priority.

The remote access to a shared place is made by means of messages in protocol RT-WMP as explained later.

The Real-time Wireless Multi-hop Protocol (RT-WMP) is a protocol for MANETs. It was designed to work over the 802.11 protocol and supports real-time traffic. In fact, end-to-end message delay in RT-WMP has a bounded and known duration and the protocol also manages global static message priorities. Besides, RT-WMP supports multi-hop communications. The protocol has been designed to connect a relatively small group (10-20 units maximum) of mobile nodes. It is based on a token-passing scheme and is designed to manage rapid topology changes through the exchange of a matrix containing link quality among nodes. RT-WMP has an error recovery mechanism that can recover from certain types of errors without jeopardizing real-time behaviour and has a technique for reincorporating lost nodes.

Even if the RT-WMP has been designed to work in wireless multi-hop networks, it can work perfectly over wired Ethernet. However, the nodes belonging to the RT-WMP protocol must have a dedicated and isolated subnetwork to guarantee the real-time performances.

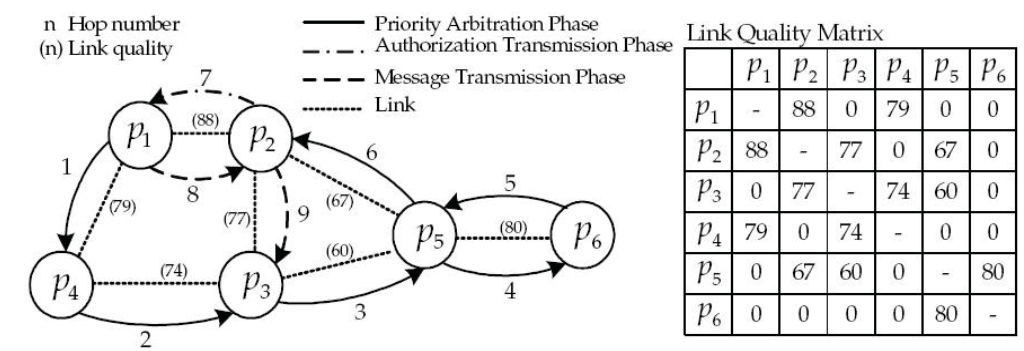

Fig. 4 A hypothetical situation described by the network graph and the corresponding LQM. The hops sequence of the protocol is also shown.

\subsection{RT-WMP Overview}

The protocol works in three phases (see Fig. 4): Priority Arbitration phase (PAP), Authorization Transmission Phase (ATP) and Message Transmission Phase (MTP). During the PAP, nodes reach a consensus over which of them holds the Most Priority Message (MPM) in the network at a given moment. Subsequently, in the ATP, an authorization to transmit is sent to the node which holds the highest priority message. Finally, in the MTP, this node sends the message to the destination node. To reach a consensus over which node holds the highest priority message, in the PAP a token travels through all of the nodes. The token holds information on the priority level of the MPM in the network and its owner among the set of nodes already reached by the token. The node which initiates the PAP states that the highest priority message in its own queue is the MPM in the whole network and stores this information in the token. Then it sends the token to another node, which checks the messages in its own queue. If the node verifies that it holds a message with a 
higher priority level than the one carried by the token, it modifies the token data and continues the phase. The last node to receive the token, which knows the identity of the MPM holder, closes the PAP and initiates the ATP. In this phase, the node calculates a path to the MPM holder using the topology information shared among the members of the network (the Link Quality Matrix, see below) and sends an authorization message to the first node in the path. The latter will route the message to the second node in the path and so on, until the authorization reaches the MPM holder. This is when the MTP begins. The development of this phase is quite similar to the preceding one. The node that has received the authorization calculates the path to reach the destination, and sends the message to the first node of the path. The message follows the path and eventually reaches its destination. The phases repeat one after another i.e., when the MTP finishes, the node destination of the message initiates a new PAP and so on. When none of the nodes have a message to transmit, the authorization and message transmission phases are omitted and the priority arbitration phases repeat continuously.

\subsection{The Link Quality Matrix}

To describe the topology of the network, RT-WMP defines an extension of the network connectivity graph (as defined in (Facchinetti, Buttazzo, et al. (2005)) adding nonnegative values on the edges of the graph. These values are calculated as functions of the radio signal between pairs of nodes and are indicators of link quality between them. These values are represented in a matrix called the Link Quality Matrix (LQM), the elements of which describe link quality between nodes (see Fig. 4). Each column describes the links of a node with its neighbours. Nodes use this matrix to select which node to pass the token to and to take decisions on the best path to route a message from a source to a destination. All the nodes have a local copy of the LQM that is updated each time a frame is received.

Besides, every node is responsible for updating its column of the LQM (both in the local copy and the shared copy) to inform the other nodes about local topology changes.

\subsection{Error Handling}

RT-WMP is quite robust in case of node failure. The implicit acknowledgement technique used dispenses with the necessity of monitoring nodes to control the loss of the token. In RT-WMP, in fact, when a $p k$ node sends a frame of any type, it listens to the channel for a timeout. The receiver $p l$ node immediately processes the frame received and sends another frame to a third $\mathrm{pm}$ node. The first sender listens to such a frame as well and interprets it as an acknowledgement. If the first sender does not hear the frame within timeout, it supposes that the $p l$ node has fallen or is out of its coverage range. In this case, the behaviour depends on the phase that the protocol is in. If it is in the ATP or MTP, pk discards the frame and starts a new PAP. However, if it is in the PAP, the $p k$ node sends the token to another node to continue the PAP without jeopardizing the temporizations (see (Tardioli and Villarroel 2007) for details). Communication errors can produce another type of problem. Let us consider the situation where, in the PAP, the $p k$ node sends a token to the pa node and waits for an implicit acknowledgment. Node pa processes the frame and sends the frame to node $p b$. As explained earlier, the last pass is also the acknowledgement for pk. However, if node $p b$ hears the frame but $p k$ does not, a token duplication occurs. In fact both nodes $p k$ and $p b$ continue the PAP and at that moment there are two tokens in the network. This problem 
was solved introducing a serial field in the frames. In this way if a node receives frames with old serials, it discards them and informs the sender sending drop-frame information.

\subsection{Use of RT-WMP}

The RT-WMP is written in C language. However, we have developed an interface Java to C with Java Native interface technology (JNI). A dynamic library has been created and the procedures have been adapted to be used from Java using the JNI.

Fig. 8 shows the access from the coordinators executed in the virtual machines of PC1 and PC3 to a shared place implemented in the virtual machine of the PC2. The access is made locally to the CPRI. The calls to the methods of CPRI are transformed into messages in protocol RT-WMP. These messages when arriving at the PC2 involve the execution of methods of the communication place.

Fig. 5 shows the Unified Modelling Language (UML) Diagram of the classes written for RTWMP communication. Two classes to represent the communication places have been implemented:

- CommunicationplaceRtWmp: for representation of the local instance of the object for which we want to invoke the methods.

- CommunicationplaceRIRtWmp: which is the remote interface of the Communicationplace.

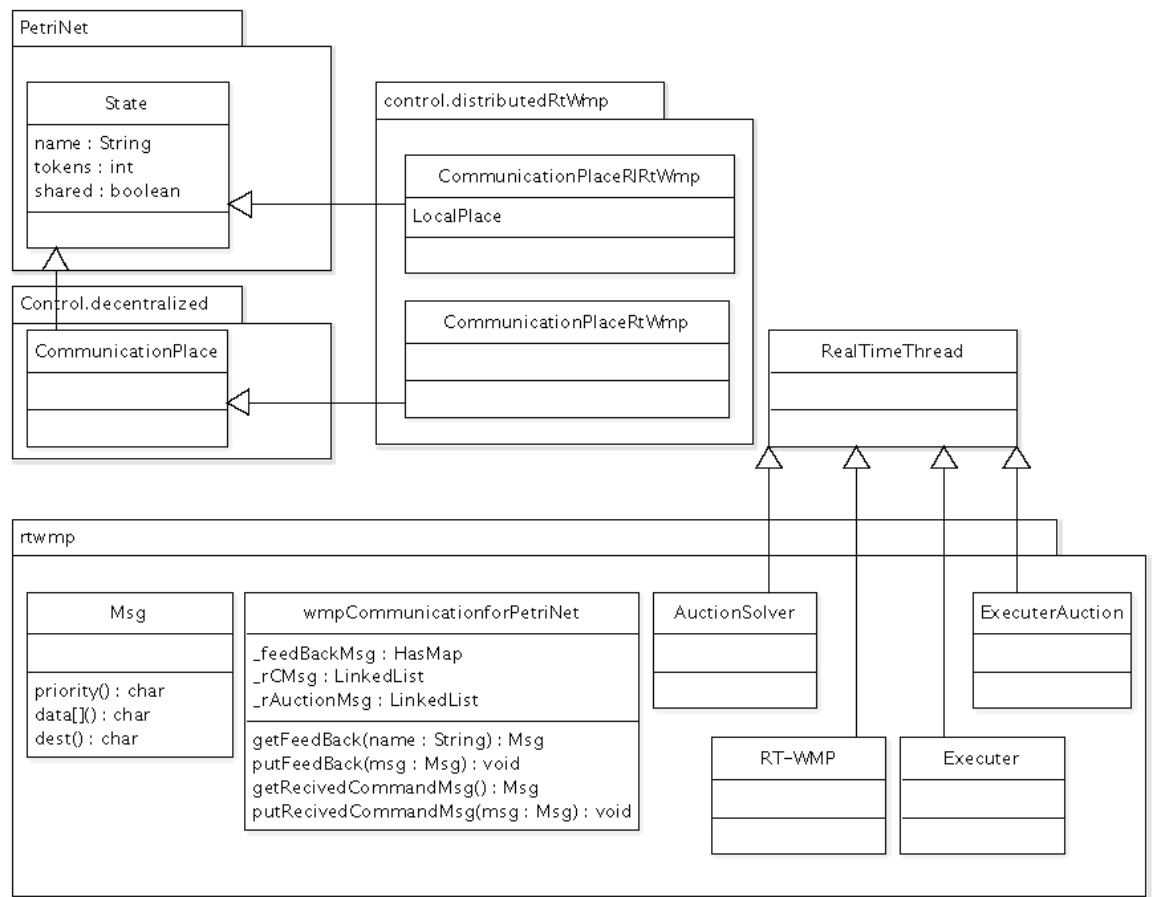

Fig. 5. RT-WMP communication Java classes. 
The threads in charge of managing the communication and the execution of the remote calls are:

- $\quad$ RT-WMP. A Real-time Thread of high priority, it manages the communication in the real-time protocol.

- Executer. A Real-time Thread in charge of executing the remote requests.

- ExecuterAuction. Similar to Executer but specialized for the tobidup () remote method because that method needs more time for its execution.

- Auction Solver. A Real-time Thread in charge of solving conflicts.

It is necessary that the tasks in charge of managing the communication are executed at higher priority than the coordinators. In order to obtain this:

- Access to the synchronized methods of the communication places is made by means of the priority ceiling protocol.

- The execution of the Auction Solver is made with a higher priority than the coordinators.

- The execution of threads that deal with the communication has a priority higher than the coordinator threads.

- Threads in charge of executing the remote procedures are executed at a priority higher than the coordinators.

Thread RT-WMP is executed at the maximum real-time priority (in our application, 38). Threads Executer, ExecuterAuction and Auction Solver are executed with a lower priority than the RT-WMP threads but greater than the Coordinators (in our application, 35). With this communication architecture a bounded response time in communication is obtained. Fig. 6 shows the Execution priorities of the threads in the proposed architecture.

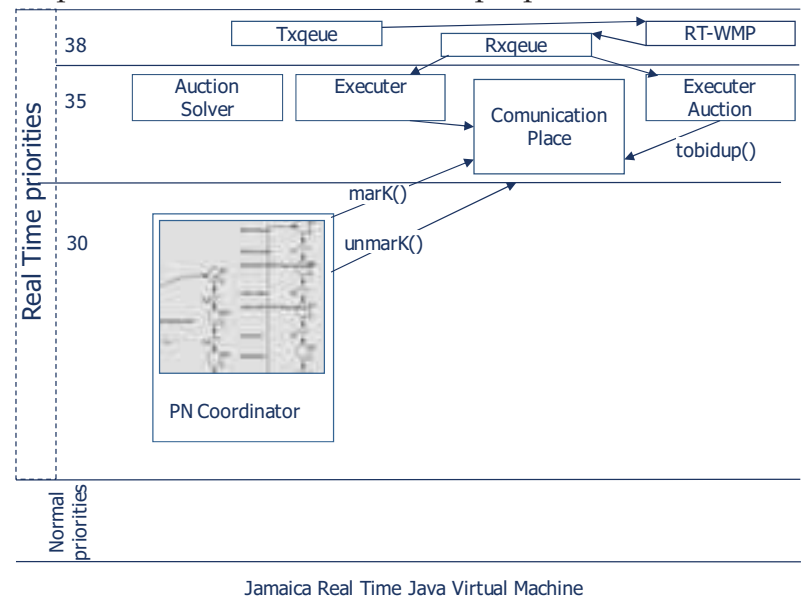

Fig. 6. Execution Priorities

\section{Distributed Control of the Flexible Manufacturing Cell}

The practical application of the technologies presented in this paper were tested using a flexible manufacturing cell installed at the Department of Computer Science and Systems Engineering at the University of Zaragoza, for research and teaching purposes. The 
manufacturing cell is composed of a set of stations for the production and storage of pneumatic cylinders.

The initial control system of the cell consisted of a programmable logic controller (PLC) that controlled each station. A real-time industrial net connected all of the PLCs.

We have installed a new distributed control system based on several computers, connected by communications networks, and a fieldbus (see Fig. 7). One of the computers is equipped with a fieldbus master card, where the input/output modules of the machines are connected. The rest of the computers control the input/output hardware by Industrial Ethernet.

Stations 1 and 4 have Inline modules (Phoenix_Contact 2006) with protocol Interbus, the master of the bus being a Computer (PC 1) with the CIF50-IBM card of Hilscher (Hilscher 2007). In the rest of the cell, Industrial Ethernet is supported. In Stations 2, 3 and in the transport, the Advantys (Schneider_Electric. 2006) input/output modules communicate in the protocol Modbus TCP over Ethernet. For Ethernet communication two segmented subnetworks are arranged. The first one is used for communication with input/output modules and the second one for real-time communication among computers in RT-WMP protocol.

Each station of the cell has a read/write head of the pallet memory that is connected to a product identifier module (Pepperl\&Fuchs IVI-KHD2-4HRX), (Pepperl\&fuchs 2006). The product identifier module is a resource shared by all stations. Communication with the identifier module is achieved using a serial port. Access to the module must be protected from concurrent access.

In our implementations, we used the Real-time Java Virtual Machine JamaicaVM v2.7 (Aicas, 2007). The target hardware was four computers with Pentium IV processors at 1.7 $\mathrm{GHz}$, running Red Hat Linux 2.4.

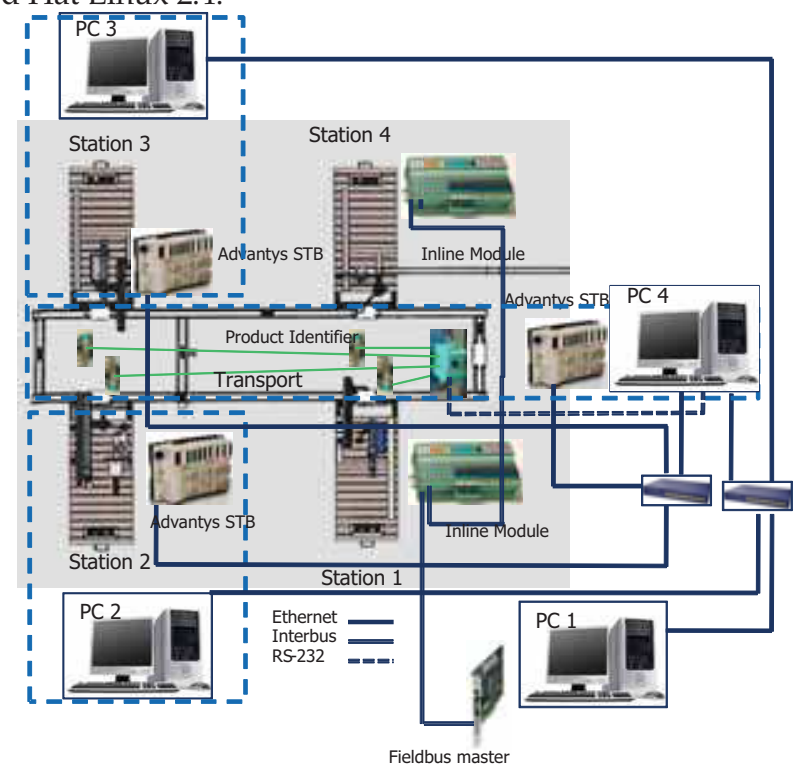

Fig. 7. Hardware Architecture 


\subsection{Software Control Architecture}

The PN that models the desired behavior of the cell has been subdivided into several subnets by following local access to the input/output modules criterion, and the local control of the machines criterion. This subdivision leads to a set of subnets and to a set of shared places that model the communications. The subnets have been implemented in several computers. Each computer is in charge of control of a part of the Cell. In each computer one or more coordinators are in charge of executing the corresponding subnet (see Fig. 8).

The execution of the coordinator takes place in a high priority thread within the Jamaica Real-Time Java Virtual Machine. PNs communicate by means of shared places (i.e. the communication places). For example, the access to the product identifier is modeled by means of a shared place that is implemented as a local communication place in the PN executed by the Cell Coordinator and as a remote communication place in the rest of the PNs.

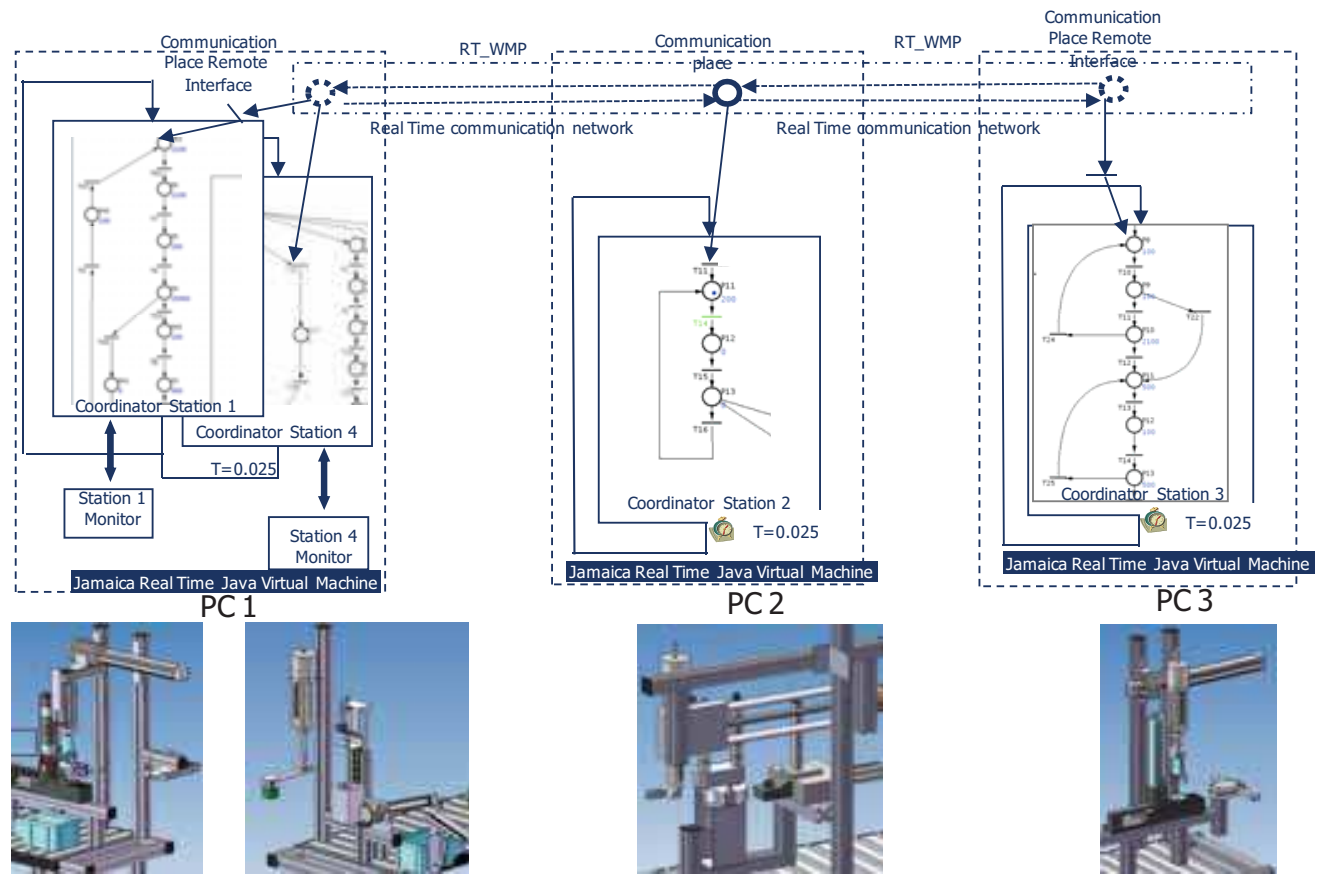

Fig. 8. Software Control Architecture

\subsection{Communication Control Architecture}

Fig. 9 shows the UML sequence diagram (Douglass 2004) of the tobidup() communication and remote execution. When in the execution of a coordinator it is desired to unmark a communication place, the method tobidup () of the CommunicationplaceRIRtWmp is called. (?)This creates a message and puts it in the transmission queue of the RT-WMP. This message is received by the RT-WMP of another node. The Executer reads the message, 
searches the corresponding CommunicationplaceRtWmp and executes the request method. Finally it sends a feedback message to the CommunicationplaceRIRtWmp. The time necessary to unmark a communication place from a remote machine is between 10 and $12 \mathrm{~ms}$.

\subsection{Control deadlines}

We have also tested the communication time among computers and the input/output modules in industrial Ethernet. This time is between 6 and $7 \mathrm{~ms}$. The data transmission time in the Interbus is deterministic and can be known using the characteristics that the manufacturer provides. In this case the data transmission time is $1.6 \mathrm{~ms}$.

With the communication times indicated, a control period of almost $25 \mathrm{~ms}$ is possible. This control period is the period of the coordinators (periodic Real-time Threads). 


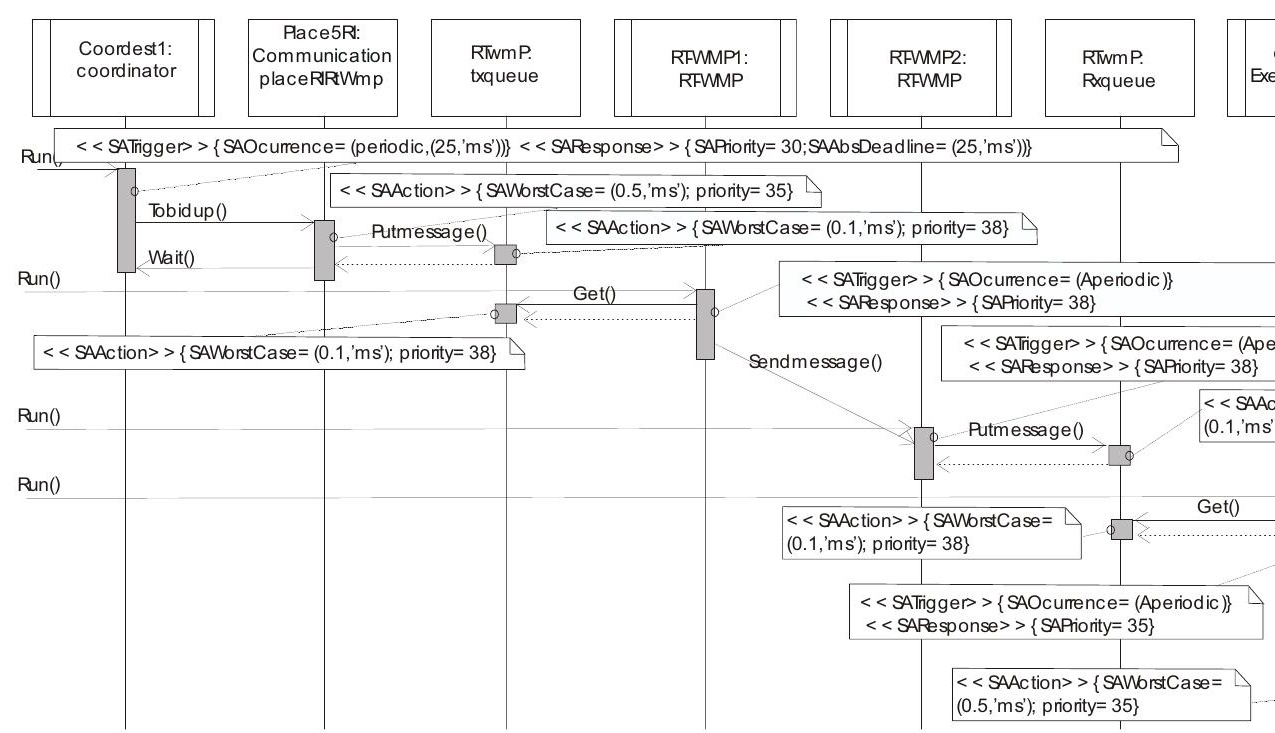

Fig. 9. Communication UML sequence diagram 
The proposed concurrent structure and priorities guarantee that the controllers execute forever using updated input data and allow real-time analysis of the thread set. Following a rate monotonic approach, all of the local controller's threads run within their period (the control period) if:

$$
C_{\text {coord }}+t_{b u s}+t_{c o m}<=T
$$

Where $T$ is the control period, $C_{\text {coord }}$ is the WCET of the local(s) coordinator(s), $t_{b u s}$ is the WCET for reading the inputs and writing the outputs in the fieldbus, $t_{\text {com }}$ is the WCET for communicating with a remote communication place.

The WCET $t_{b u s}$ in the fieldbus Ethernet is $7 \mathrm{~ms}$. In the bus Interbus it is $1.6 \mathrm{~ms}$. The WCET for communication is $12 \mathrm{~ms}$. In the PC1(fieldbus Interbus):

$$
C_{\text {coordest } 1}+C_{\text {coordest } 4}+t_{\text {bus }}+t_{\text {com }}=1+1+1.6+12=15.6<=25 \mathrm{~ms} \text {. }
$$

In the PC2, PC3 and PC4 (fieldbus Ethernet):

$$
\begin{aligned}
& C_{\text {coordest } 2}+t_{\text {bus }}+t_{\text {com }}=1.2+7+12=20.2<=25 \mathrm{~ms} .(P C 2) \\
& C_{\text {coordest } 3}+t_{\text {bus }}+t_{\text {com }}=1.1+7+12=20.1<=25 \mathrm{~ms} .(\text { PC } 3) \\
& C_{\text {coordtransport }}+t_{\text {bus }}+t_{\text {com }}=0.9+7+12=19.9<=25 \mathrm{~ms} .(\text { PC } 4)
\end{aligned}
$$

If the previous condition is fulfilled, the worst-case response time for events in the system can be calculated as:

$$
t_{r} \leq 2 T+t_{\text {bus }}
$$

That is, the response time $\left(t_{r}\right)$ has a bound related to the control period $(T)$ and the readingwriting time of the bus $\left(t_{b u s}\right)$. An example of system response time to an incoming event is presented in Fig. 10

In our application, the control period is $25 \mathrm{~ms}$, enough for the dynamics of the controlled system. With Ethernet, the maximum read-write time is $7 \mathrm{~ms}$; therefore, the response time of the real-time control will be:

$$
t_{r} \leq 2 * T+t_{\text {bus }}=2 * 0.025+0.007=0.057 \mathrm{~s}
$$




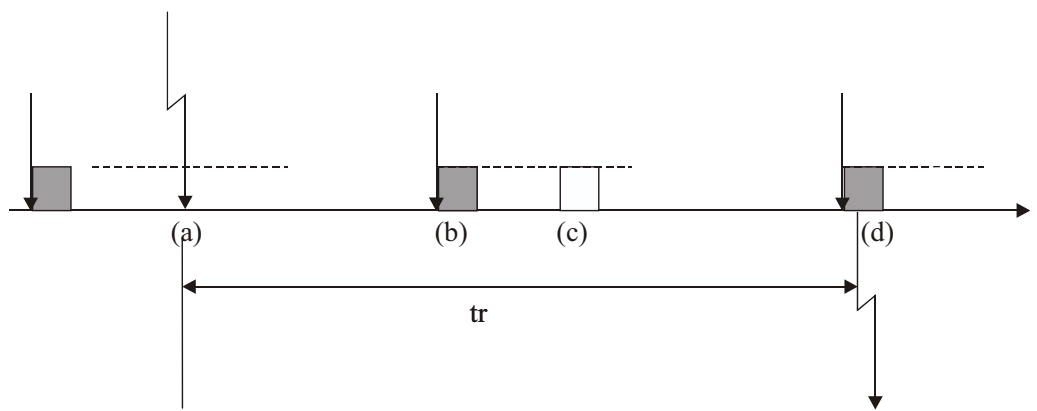

Fig. 10. System control time response:

a) An event happens in the system (e.g., a pallet arrives at a station).

b) The event is copied to the memory image of the control system.

c) A local controller reads the event and establishes the reaction as changes on the memory image of outputs.

d) The outputs are established in the system through the fieldbus.

\section{Conclusions}

In this paper a new PN implementation technique called distributed coordinators has been developed. This technique involves the use of centralized, decentralized and distributed implementations.

A platform that allows the implementation of distributed PNs has been developed. For this purpose, a set of Java Classes has been designed to implement synchronous and asynchronous communication paradigms. These classes implement the communications places and their remote interfaces: communications places remote interface, the auction communication place and the Auction solver. We propose a method to implement simple conflicts in distributed PN. In future works we intend to study multiple and coupled conflicts.

To grant the real-time execution of the remote calls to the methods of the remote communication places, a new software architecture has been implemented on top of the real-time protocol RT-WMP. In this way the system is analyzable from the real-time perspective.

All of the techniques and technologies presented in this work have been evaluated in a practical application: the distributed control of a flexible manufacturing cell. The control system of the cell is currently running without problems. This work, which is an extension of our previous research, provides the basis for further investigations into PN software implementations, languages, and real-time execution platforms.

\section{References}

Aicas, G. (2007). JamaicaVM Realtime Java Technology. http://www.aicas.com/.

Bollella, G. and J. Gosling ( 2000). "The Real-time Specification for Java." Computer 33(6): 4754 . 
Borg, A. and A. Wellings (2003). A real-time RMI framework for the RTSJ. 15th Euromicro Conference on Real-time Systems (ECRTS'03).

Brams, G. V. (1982). Reseaux de Petri: Theorie et Practique, Vols. I and II, Masson.

Briz, J. L. and J. M. Colom (1994). "Implementation of Weighted Place/Transition Nets based on Linear Enabling Functions." Application and Theory of Petri nets 815: 99-118.

Bruno, G. and G. Marchetto (1986). "Process-translatable Petri nets for the rapid Prototyping of Process-Control Systems." Ieee Transactions on Software Engineering 12(2): 346357.

Colom, J. M., M. Silva, et al. (1986). "On software implementation of Petri nets and colored Petri nets using high-level concurrent languages." Seventh European Workshop on applications and theory of Petri nets, Oxford, July 86: 207-241.

Douglass, B. P. (2004). Real-time UML: advances in the UML for real-time systems, Addison Wesley Longman Publishing Co., Inc. Redwood City, CA, USA.

Facchinetti, T., G. Buttazzo, et al. (2005). Resource reservation and connectivity tracking to support real-time communication among mobile units. EURASIP J. Wirel. Commun. Netw., 5(5):712-730, 2005.

García, F. J. and J. L. Villarroel (1998). Decentralized Implementation of Real-Time Systems Using Time Petri nets. Application to Mobile Robot Control. 5th IFAC Workshop on Algorithms and Architectures for Real-time Control Cancun, Mexico.

Hilscher. (2007). "PC cards. http:/ / www.hilscher.com."

Martínez, J. M., M. G. Harbour, et al. (2003). RT-EP: Real-Time Ethernet Protocol for Analyzable distributed Applications on a Minimum Real-Time POSIX Kernel.

Murata, T. (1989). "Petri nets- Properties, Analysys and applications." Proceedings of the Ieee 77(4): 541-580.

Pepperl\&fuchs. (2006). "IVI-KHD2-4HRX DataSheet. http:/ / www.pepperl-fuchs.com." 2006.

Phoenix_Contact. (2006). "Inline Modules. www.phoenixcontact.com. ."

Piedrafita, R. and J. L. Villarroel (2006 a). Implementation of time Petri nets in real-time Java. Proceedings of the 4th international workshop on Java technologies for real-time and embedded systems.

Piedrafita, R. and J. L. Villarroel (2006 b). Petri nets and Java. Real-Time Control of a flexible manufacturing cell. Emerging Technologies and Factory Automation, 2006. ETFA'06. IEEE Conference on.

Schneider_Electric. (2006). "Advantys STB. http:/ / www.telemecanique.com."

Tardioli, D. and J. L. Villarroel (2007). Real-time communications over 802.11: RT-WMP.

Taubner, D. (1988). "On the implementation of Petri nets." Lecture Notes in Computer Science 340: 418-439.

Villarroel, J. L. (1990). Integración Informática del Control de Sistemas Flexibles de Fabricación. Tesis Doctoral. Ingeniería Eléctrica e Informática, Universidad de Zaragoza. 


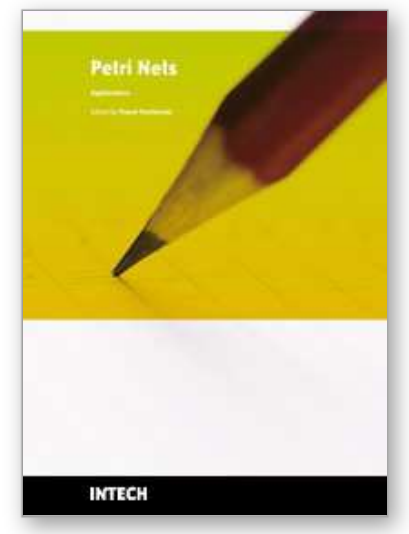

\author{
Petri Nets Applications \\ Edited by Pawel Pawlewski
}

ISBN 978-953-307-047-6

Hard cover, 752 pages

Publisher InTech

Published online 01, February, 2010

Published in print edition February, 2010

Petri Nets are graphical and mathematical tool used in many different science domains. Their characteristic features are the intuitive graphical modeling language and advanced formal analysis method. The concurrence of performed actions is the natural phenomenon due to which Petri Nets are perceived as mathematical tool for modeling concurrent systems. The nets whose model was extended with the time model can be applied in modeling real-time systems. Petri Nets were introduced in the doctoral dissertation by K.A. Petri, titled “"Kommunikation mit Automaten” and published in 1962 by University of Bonn. During more than 40 years of development of this theory, many different classes were formed and the scope of applications was extended. Depending on particular needs, the net definition was changed and adjusted to the considered problem. The unusual "flexibility" of this theory makes it possible to introduce all these modifications. Owing to varied currently known net classes, it is relatively easy to find a proper class for the specific application. The present monograph shows the whole spectrum of Petri Nets applications, from classic applications (to which the theory is specially dedicated) like computer science and control systems, through fault diagnosis, manufacturing, power systems, traffic systems, transport and down to Web applications. At the same time, the publication describes the diversity of investigations performed with use of Petri Nets in science centers all over the world.

\title{
How to reference
}

In order to correctly reference this scholarly work, feel free to copy and paste the following:

Ramon Piedrafita, Danilo Tardioli and Jose Luis Villarroel (2010). Distributed Implementation of Petri nets in Control Applications, Petri Nets Applications, Pawel Pawlewski (Ed.), ISBN: 978-953-307-047-6, InTech, Available from: http://www.intechopen.com/books/petri-nets-applications/distributed-implementation-of-petrinets-in-control-applications

\section{INTECH}

open science | open minds

\section{InTech Europe}

University Campus STeP Ri

Slavka Krautzeka 83/A

51000 Rijeka, Croatia

Phone: +385 (51) 770447

Fax: +385 (51) 686166

www.intechopen.com

\section{InTech China}

Unit 405, Office Block, Hotel Equatorial Shanghai

No.65, Yan An Road (West), Shanghai, 200040, China 中国上海市延安西路65号上海国际贵都大饭店办公楼405单元

Phone: +86-21-62489820

Fax: $+86-21-62489821$ 
(C) 2010 The Author(s). Licensee IntechOpen. This chapter is distributed under the terms of the Creative Commons Attribution-NonCommercial-ShareAlike-3.0 License, which permits use, distribution and reproduction for non-commercial purposes, provided the original is properly cited and derivative works building on this content are distributed under the same license. 